\title{
On phytochrome absorption and the phytochrome photoequilibrium in a green leaf: environmental sensitivity and photoequilibrium time
}

\author{
Andrea Rivadossi, Flavio M. Garlaschi, Anna Paola Casazza, Giuseppe Zucchelli and Robert C. Jennings* \\ Received 11th April 2008, Accepted 12th June 2008 \\ First published as an Advance Article on the web 25th June 2008 \\ DOI: 10.1039/b806142d
}

The average, corrected attenuance spectra for both spectral forms of phytochrome in a mature leaf were calculated. Optical masking by chlorophyll together with the detour effect (optical path lengthening effect) due to multiple light scattering led to large changes in both the $\mathrm{Q}_{\mathrm{y}}$ band shape and wavelength position and the effective intensity of the weak vibrational bands increases. The $\mathrm{Pfr} / \mathrm{Pr}$ oscillator-strength-ratio between $400-750 \mathrm{~nm}$ (0.93 in vitro), becomes 1.63 in a leaf. Thus the dominant absorption form is Pfr. These two values permit calculation of the phytochrome photoequilibrium under conditions of "daylight" illumination both in vitro and in folia. These values are 0.6 and 0.38 respectively. Previous literature estimates for the situation in vitro, based on the $660 / 730 \mathrm{~nm}$ absorption ratio, yielded values close to 0.6 . It is demonstrated that this large decrease in the phytochrome photoequilibrium in a leaf has the effect of translating this parameter to a position on the dose (red/far-red light ratio)-response (Pfr/Ptot) plot towards greater sensitivity to changes in the environmental red/far-red ratio. The increased sensitivity factor is almost five-fold for the "daylight" environment and is even greater for the various "shade-light" environments. The approximate time taken to attain photoequilibrium (1/e lifetime) has also been calculated for phytochrome in a leaf in different light environments. For the "daylight" environment the photoequilibration time is $\sim 5 \mathrm{~s}$, which increases into the 20-80 s interval under different degrees of "shade light". Thus, despite the strong optical masking by chlorophyll in a mature leaf, the phytochrome photoequilibrium is attained quite rapidly on a physiological time scale.

\section{Introduction}

The phytochromes are an ubiquitous family of pigment/protein plant signal transducing photoreceptors which, on the basis of their unique capacity to sense the light environment, constitute the principle system by which plants are able to determine position and time. ${ }^{1,2}$ Under sun light illumination it is well known that phytochrome cycles between two distinct spectroscopic forms due to their striking property of reversible photochromism. Upon light absorption the lowest lying singlet excited state $\left(\mathrm{Q}_{\mathrm{y}}\right)$, which is also the most intensely absorbing transition, undergoes an absorption band shift of about $70 \mathrm{~nm}$ towards the red, thought to be largely due to a cis-trans isomerisation around the $\mathrm{C}_{15}$ bond ${ }^{3}$ of the noncyclic tetrapyrrole chromophore. The blue-most absorbing form, known as $\mathrm{Pr}$, has its $\mathrm{Q}_{\mathrm{y}}$ absorption maximum near $660 \mathrm{~nm}$, and this is the form which is synthesised. The long wavelength form, known as Pfr, has its $\mathrm{Q}_{\mathrm{y}}$ absorption maximum near $730 \mathrm{~nm}$ and this is the form usually considered to be physiologically active. Phytochrome is normally present at very low concentrations in green, chlorophyll containing tissues, and this leads to optical masking by chlorophylls, present at very much greater concentrations in leaves. ${ }^{4}$ This prevents in vivo spectroscopic studies on light grown tissue and it has been suggested that this is the reason why phytochrome action spectra are blue shifted with respect to Pr absorption. ${ }^{4,5}$ In this respect it should be noted that the optical

Istituto di Biofisica del Consiglio Nazionale delle Ricerche, Sede di Milano, Dipartimento di Biologia, Università degli Studi di Milano, via Celoria 26, 20133, Milano, Italy.E-mail: robert.jennings@unimi.it masking by chlorophylls will not be equal for the two phytochrome forms. This is due to chlorophyll absorption in leaves tailing off at wavelengths above $700 \mathrm{~nm} .{ }^{6,7}$ Thus chlorophyll masking is expected to be greater for Pr than for Pfr, and this is implicit in the photoequilibrium calculations of Holmes and Fukshansky. ${ }^{8}$ In addition, within a leaf multiple light scattering leads to a light environment which is essentially diffuse in nature and this, in turn, will modify light absorption by the two phytochrome forms in a wavelength dependent way, by virtue of the so called "detour effect" ${ }^{6,9}$ sometimes also called the optical path lengthening effect. It is therefore of interest to understand just what the corrected attenuance characteristics of phytochrome in green tissue are and, as this has not yet been determined, as far as we are aware, we have analysed the problem. In addition, this has allowed us to determine the average phytochrome photoequilibrium in a mature leaf, which is significantly less than the solution ratio. It is furthermore demonstrated that this significant decrease of the $\mathrm{Pfr} /(\mathrm{Pr}+\mathrm{Pfr})$ ratio in a leaf leads to an almost five fold increase in the sensitivity of the phytochrome system to the red/far-red light environment. The present analysis is largely based on existing experimental data in the literature, and takes advantage of an approach which we have developed for examining the in folia absorption of chlorophyllprotein complexes. ${ }^{7,10}$

\section{Materials and methods}

Thylakoids were extracted from freshly harvested spinach leaves as described by Garlaschi et al. ${ }^{6}$ The thylakoid absorption spectrum 
was measured with a Jasco Uvidec 510 spectrophotometer, using an opal glass to minimize light scattering, in the wavelength range $400-760 \mathrm{~nm}$.

Leaf transmittance and reflectance spectra were measured in a Jasco Uvidec 510 spectrophotometer equipped with an integration sphere (TIS 314) as described by Garlaschi et al. ${ }^{6}$ The leaf absorption spectra, attenuance and $(1-T)$, were determined taking into account the reflectance correction. The phytochrome absorption spectra were taken from the published data of Lagarias et al. ${ }^{11}$ Spectral decomposition of the $\mathrm{Q}_{\mathrm{y}}$ absorption region was performed with a minimum number of asymmetric Gaussian sub-bands (four) in order to approximately quantitate the electronic/vibrational band contributions. The software used an Excel solver to perform the minimisation. The goodness of the fit was based on the $\chi^{2}$ value and the residuals distribution.

\section{Results and discussion}

In the following we use the absorption spectra for both the Pr and Pfr forms of native, solubilised phytochrome published by Lagarias et al. ${ }^{11}$ as these are amongst the clearest available. We assume that the multiple phytochrome gene products (PHYA, PHYB, PHYC, PHYD, PHYE) have similar optical properties to the PHYA studied by Lagarias $e t$ al. ${ }^{11}$ This assumption may be supported by the in vitro reconstituted phytochromes A, B, $\mathrm{C}$, and $\mathrm{E}$ dark minus light difference spectra ${ }^{12}$ where only small spectroscopic variations were observed. The optical absorption spectra for Pr and Pfr are shown in Fig. 1. As is well known, there are two principal regions of phytochrome absorption in the $\mathrm{red} / \mathrm{far}$-red and in the blue, with over $80 \%$ of the total oscillator strength associated with the red/far-red transitions. These long wavelength transitions are very broad with the $\mathrm{Q}_{\mathrm{y}}$ electronic transition maxima near $660 \mathrm{~nm}(\mathrm{Pr})$ and $730 \mathrm{~nm}$ (Pfr). Each phytochrome form has clear vibrational structure in the $\mathrm{Q}_{\mathrm{y}}$ region, giving rise to an absorption shoulder about $50 \mathrm{~nm}$ to the blue of the $\mathrm{Q}_{\mathrm{y}}$ maxima. In order to estimate the vibrational/electronic absorption contributions (v/e ratio) the spectra were subjected to a Gaussian sub-band decomposition analysis (Fig. 2). Using a minimum number of four (asymmetric) Gaussian sub-bands we find that $\mathrm{v} / \mathrm{e}=0.5$ for $\mathrm{Q}_{\mathrm{y}}$ absorption in both Pr and Pfr. Thus, it is the broad band associated with the electronic transitions, in both Pr and Pfr, that accounts for most absorption in vitro. We underline this point as it will be demonstrated below that, with respect to

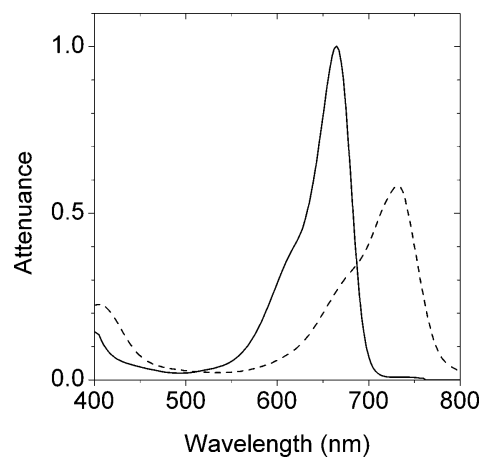

Fig. 1 Optical attenuance spectra of the red (Pr; solid line) and far-red (Pfr; dashed line) absorbing forms of native oat phytochrome. The spectra have been taken from Lagarias et al. ${ }^{11}$

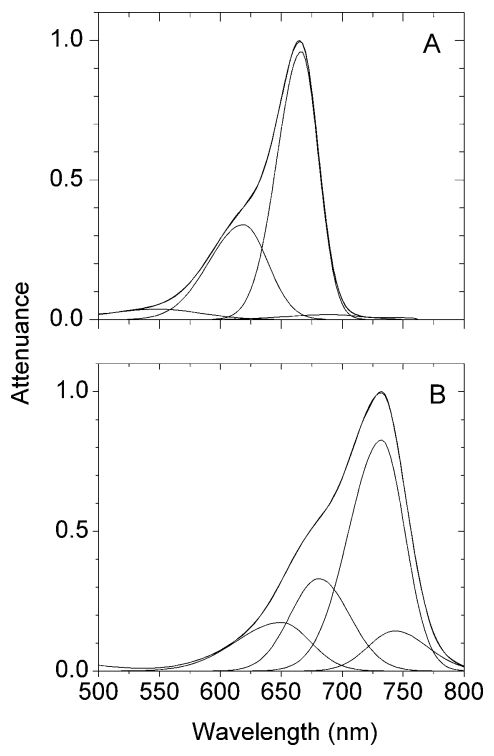

Fig. 2 Gaussian sub-band decomposition of the $\mathrm{Q}_{\mathrm{y}}$ absorption region of the Pr (panel A) and Pfr (panel B) spectra taken from Lagarias et al. ${ }^{11}$ Both measured spectra and the resulting fits are shown.

this solution value of $\mathrm{v} / \mathrm{e}=0.5$, the vibrational contribution to the corrected attenuance spectrum of $\mathrm{Pr}$ in a leaf is considerably greater $(\mathrm{v} / \mathrm{e}=1)$, while that for $\mathrm{Pfr}$ is considerably less $(\mathrm{v} / \mathrm{e}=0.1)$.

The absorption spectra of isolated phytochrome (Fig. 1) do not, however, tell us very much about the details of light absorption under physiological conditions, i.e. for example in a leaf, as these are expected to differ considerably from that of the isolated complexes due to optical phenomena associated with the leaf such as the detour effect as well as filtering effects due to chlorophyll. ${ }^{6,8-10}$ In the present study we employ the absorption spectra of Fig. 1 in order to determine an average corrected attenuance spectra for Pr and Pfr in the chlorophyll-containing and multiple scattering environment of a mature spinach leaf. The approach used is based on the previous analysis of the corrected attenuance spectrum of pigment-protein complexes in leaves. ${ }^{7,10}$

The starting point was the demonstration that the attenuance spectrum (absorption) of a chloroplast suspension could be accurately described by the weighted linear sum of the absorption spectra of isolated PSI, PSII and LHCII. The linear sum PSI + PSII + LHCII spectrum is then transformed into a leaf absorption spectrum (attenuance) by means of an empirically derived "detour effect" correction spectrum $(\Delta(\lambda)$; eqn (1)). This "detour effect" correction spectrum is based on the optical density ratio of the leaf spectrum $\left(A_{\mathrm{L}}\right)$ with respect to the chloroplast spectrum $\left(A_{\mathrm{C}}\right)$ at the same chlorophyll concentration per unit area. The "detour effect" is due to multiple scattering inside the leaf and is caused by the different refractive indices at the water-air interface ${ }^{9,6}$ due to the presence of intercellular air spaces. It increases the optical path length of light within the leaf in a way which is strongly wavelength dependent and is expected to be similar for all chromophores within the diffuse-light environment of a leaf. The empirically derived correction spectrum $(\Delta(\lambda))$ is presented in Fig. 3 and is maximal between $530-650 \mathrm{~nm}$ and above $700 \mathrm{~nm}$. It is minimal near $440 \mathrm{~nm}$ and $680 \mathrm{~nm}$ where chl $a$ absorption is dominant. The leaf attenuance spectrum is given in the exponent in eqn (1), 


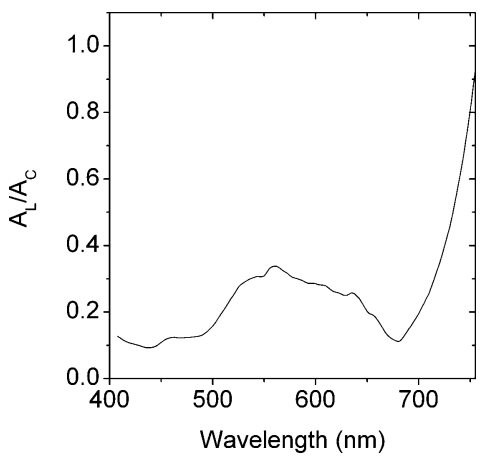

Fig. 3 The leaf "detour effect" spectrum $[\Delta(\lambda)]$ determined from the experimental leaf and chloroplast absorption spectra $\left(A_{\mathrm{L}} / A_{\mathrm{C}}\right)$ measured at the same chlorophyll concentration.

written for the measured photon absorption spectrum of the leaf $\left[(1-T)_{\mathrm{L}}(\lambda)\right]$,

$$
(1-T)_{\mathrm{L}}(\lambda) \cong 1-10^{-[a \operatorname{PSI}(\lambda)+\beta \operatorname{PSII}(\lambda)+\delta \operatorname{LHCII}(\lambda)] \Delta(\lambda)}
$$

where $\alpha, \beta$, and $\delta$ are weighting factors which have no specific physical meaning and are determined by the fitting procedure used to describe the chloroplast absorption (attenuance) spectrum; the subscript $\mathrm{L}$ refers to leaf. Owing to the extremely low concentrations of phytochrome $\left(3 \times 10^{12}\right.$ molecules $\left.\mathrm{cm}^{-2}\right)$, based on the monoclonal antibody determinations of Sharrock and Clack,$^{13}$ which is less than $10^{-4}$ times that of chlorophyll in a mature leaf $\left(30 \mu \mathrm{g} \mathrm{chl} \mathrm{cm}^{-2}=2 \times 10^{16}\right.$ molecules $\left.\mathrm{cm}^{-2}\right)$, this chromophore may be ignored at the level of defining leaf absorption and is thus not present in eqn (1).

In the linear sum approximation, ${ }^{10}$ the average corrected attenuance spectrum of phytochrome in a leaf, $\left[(1-T)_{\mathrm{P}}(\lambda)\right]$, is given by

$$
(1-T)_{\mathrm{P}}(\lambda)=\left(A_{\mathrm{P}}(\lambda) /\left(A_{\mathrm{C}}(\lambda)+A_{\mathrm{P}}(\lambda)\right)\right)(1-T)_{\mathrm{L}}(\lambda) ;
$$

$A(\lambda)$ is the absorption spectrum (attenuance) and the subscripts $\mathrm{P}, \mathrm{C}$, and $\mathrm{L}$ refer to phytochrome, chloroplasts and the leaf respectively. As $A_{\mathrm{C}}(\lambda)$ is many orders of magnitude greater than $A_{\mathrm{P}}(\lambda)$, this latter term may be dropped from the denominator on the right of eqn (2). In the following we consider the leaf to be immersed in the natural "daylight" spectral quality, which is simply included as a linear multiplicative term $E(\lambda)^{7,10}$

$$
(1-T)_{\mathrm{P}}(\lambda)=E(\lambda)\left[\left(A_{\mathrm{P}}(\lambda) / A_{\mathrm{C}}(\lambda)\right)(1-T)_{\mathrm{L}}(\lambda)\right] .
$$

In Fig. 4 the average corrected attenuance spectra are presented for Pr and Pfr in a mature spinach leaf, together with the Lagarias et al. ${ }^{11}$ optical density absorption spectra for comparison. The spectra are normalised to their respective maxima.

For $\operatorname{Pr}$ the corrected attenuance spectrum in the $\mathrm{Q}_{\mathrm{y}}$ region is substantially blue shifted in a leaf, as expected, with the broad effective absorption maximum being blue shifted by about $20 \mathrm{~nm}$. The effective absorption bandwidth is greatly increased as is evident by noting that the FWHM is $47 \mathrm{~nm}$ for the attenuance spectrum and $87 \mathrm{~nm}$ for the corrected attenuance in a leaf. This is caused by both the "detour effect", which leads to an approximate tripling of light absorption at $560 \mathrm{~nm}$ with respect to $660 \mathrm{~nm}$ (Fig. 3), and optical masking by chlorophyll that changes the spectral distribution of the light incident on phytochrome. It will also be noticed that the pronounced broadening is significantly

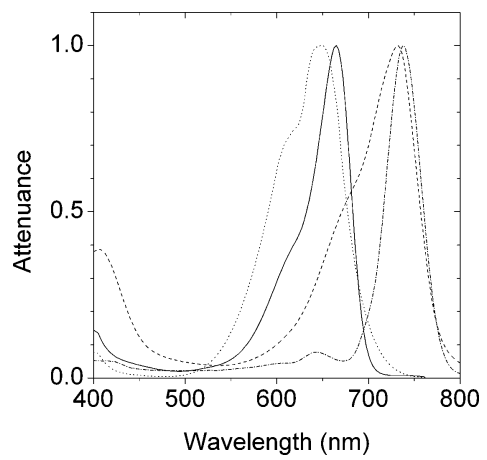

Fig. 4 Optical absorption (attenuance) spectra of $\operatorname{Pr}$ (solid line) and $\mathrm{Pfr}$ (dashed line), normalised to the peak values. These spectra have been taken from Fig. 1. Also presented are the effective average absorptance spectra calculated for Pr (dotted line) and Pfr (dash-dot line) in a mature spinach leaf and normalised to the peak values. See text for further details.

associated with a marked increase in the vibrational shoulder near $600 \mathrm{~nm}$. Spectral decomposition analysis in Gaussian sub-bands shows that the vibrational contribution to Pr in folia increases to represent about $50 \%$ of the total corrected attenuance (data not shown) and the $\mathrm{v} / \mathrm{e}$ ratio thus approaches 1 . This interesting point is not altogether surprising, as it has already been demonstrated to occur for the weak chlorophyll vibrational bands in a leaf. ${ }^{10}$ It can also be seen that the weak $400 \mathrm{~nm}$ transition is greatly decreased in the corrected attenuance spectrum and this is almost entirely due to chlorophyll masking.

For the $\mathrm{Q}_{\mathrm{y}}$ absorption of $\mathrm{Pfr}$, the optical properties of the leaf have the opposite effect with respect to Pr. The band maximum is red shifted by about $5 \mathrm{~nm}$ and a marked band narrowing is observed with the FWHM of the Pfr attenuance spectrum changing from $86 \mathrm{~nm}$ to $45 \mathrm{~nm}$ for the corrected attenuance spectrum in the leaf (Fig. 4). Whilst the pronounced band narrowing on the short wavelength side is entirely due to chlorophyll masking, it is the detour effect which increases Pfr absorption dramatically on passing from 700 to $750 \mathrm{~nm}$ (Fig. 3), and which is largely responsible for the red shift. The $\mathrm{Q}_{\mathrm{y}}$ vibrational contribution to Pfr absorption almost disappears in the leaf, as does the transition near $400 \mathrm{~nm}$.

The Lagarias et al. ${ }^{11}$ absorption spectra are for equi-molar concentrations of $\operatorname{Pr}$ and Pfr. By integration under the absorption profiles, it can be shown that the ratio ( $\mathrm{Pfr} / \mathrm{Pr})$ of the oscillator strengths between $400 \mathrm{~nm}$ and $750 \mathrm{~nm}$ is 0.93 . The average corrected leaf attenuance spectrum for equi-molar concentrations of the two phytochrome forms is shown in Fig. 5, where it is clear that, due to the $\mathrm{Q}_{\mathrm{y}}$ region, $\mathrm{Pfr}$ is dominant. The equimolar Pfr/Pr corrected attenuance ratio (AR) in folia is 1.64. This means that under normal "daylight" conditions Pfr has an equimolar absorption capacity which is about $76 \%$ greater than that of Pr based on the respective oscillator strengths. This will lead to a significant lowering in the phytochrome photoequilibrium with respect to that determined without considering leaf optical properties, in agreement with the much earlier report by Holmes and Fukshansky. ${ }^{8}$ We wish to underline this point as it has been a long and continuing practice to calculate the photoequilibrium ratio in green tissue without considering leaf optical properties. Perhaps this is based on the assumption that active phytochrome is localised only in the non-pigmented leaf epidermis. As far as 


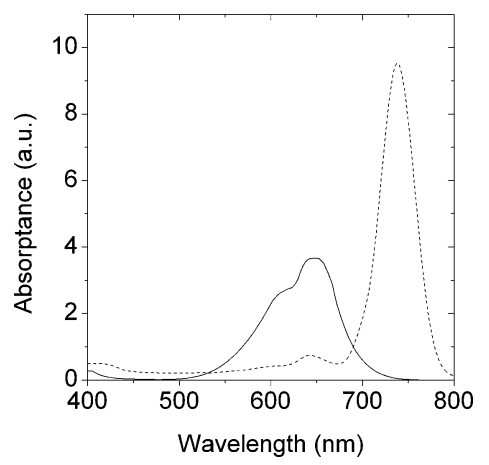

Fig. 5 The absolute, average effective absorptance spectra, of Pr (solid line) and Pfr (dashed line) in a mature spinach leaf. It can be seen that despite the lower $\mathrm{Q}_{\mathrm{y}}$ oscillator strength of Pfr it is the dominant absorbing form in a leaf.

we are aware such an assumption has never been demonstrated and, as will become evident below, this would not be particularly advantageous to the plant.

We now specifically address the question of the average phytochrome photoequilibrium, Pfr/Ptot, in a leaf, where Ptot $=\mathrm{Pfr}$ $+\operatorname{Pr}$. This average value can be compared with our determination, based on the above mentioned value of $\mathrm{AR}=1.64$, in the following way. For the simple, classical phytochrome model

$$
\stackrel{k_{s}}{\longrightarrow} \operatorname{Pr}_{\stackrel{k_{1}}{\longrightarrow}}^{\stackrel{k_{1}}{\longrightarrow}} \operatorname{Pfr} \stackrel{k_{\mathrm{d}}}{\longrightarrow}
$$

the photostationary state can be shown to be Pfr/Ptot $=k_{1}\left(k_{1}+\right.$ $\left.k_{-1}+k_{\mathrm{d}}\right)^{-1}$. The thermal degradation of $\operatorname{Pfr}\left(k_{\mathrm{d}}\right)$ seems to apply only to PHYA, which is virtually absent in light grown plants. ${ }^{14}$ Thus for the other phytochrome types $k_{\mathrm{d}}$ is thought to approximate zero. Thus Pfr/Ptot $=k_{1}\left(k_{1}+k_{-1}\right)^{-1}$ for light grown plants, which, incidentally, is the same ratio as that for the phytochrome equilibrium when the rate of synthesis of Pr is not considered.

We now assume that the photochemical conversion efficiency is equal in both directions i.e. $k_{1}=k_{-1} \cdot{ }^{4}$ As $k_{1}$ and $k_{-1}$ scale linearly with photon absorption at each wavelength $\left((1-T)_{\mathrm{p}}(\lambda)\right)$, from eqn (2) or (3) the Pfr/Ptot equals the photon absorption ratio $\operatorname{Ar}(\lambda)\left((\operatorname{Ar}(\lambda)+\operatorname{Afr}(\lambda))^{-1}\right.$, where the Ar and Afr are the photon absorption of $\operatorname{Pr}$ and Pfr. Thus $k_{1}\left(k_{1}+k_{-1}\right)^{-1}=(1+\mathrm{AR})^{-1}=0.38$ under "daylight" conditions. This average value is very close to an earlier estimate of this kind by Holmes and Fukshansky ${ }^{8}$ and is considerably lower than literature values $(\cong 0.6)$ for leaves in which the optical properties are not considered. This appreciable decrease of the photoequilibrium value in a mature leaf is expected to have a significant influence on the sensitivity of the phytochrome system to changes in the environmental light quality (red/far-red). It is well known, from the early studies of Smith and Holmes, ${ }^{15}$ that the value of the phytochrome photoequilibrium increases hyperbolically as a function of the red/far-red photon flux ratio. If we define the phytochrome sensitivity to the red/far-red light flux as the first derivative of this hyperbola $(\mathrm{d}(\mathrm{Pfr} / \mathrm{Ptot}) / \mathrm{d}(\mathrm{red} / \mathrm{far}-$ red)), it is expected that this will be greatest when the $\mathrm{Pfr} / \mathrm{Ptot}$ ratio is low. This first derivative (phytochrome sensitivity) for the Smith and Holmes ${ }^{15}$ data is shown in Fig. 6 as a function of the Pfr/Ptot ratio. The vertical arrows indicate the phytochrome sensitivity under daylight illumination for the in vitro situation, which is presumably also similar in etiolated tissue, and for the in folia situation. The decrease in the Pfr/Ptot ratio from 0.6

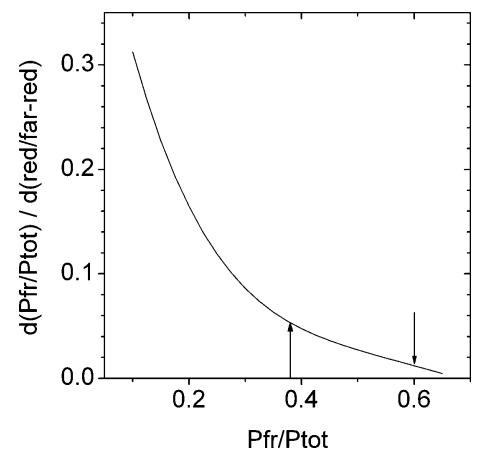

Fig. 6 Light environmental sensitivity of the phytochrome system to different red-light/far-red-light ratios as a function of the photoequilibrium. The phytochrome sensitivity parameter is the first derivative of the phytochrome photoequilibrium with respect to the red-light/far-red-light ratio $(\mathrm{d}(\mathrm{Pfr} / \mathrm{Ptot}) / \mathrm{d}(\mathrm{red} /$ far-red $))$ and was calculated from the data of Smith and Holmes. ${ }^{15}$ For further details, see text. The vertical arrow on the right hand side represent the phytochrome photoequilibrium often determined from the in vitro absorption properties of phytochrome whereas the other arrow represents the in folia situation.

to 0.38 leads to a large increase in phytochrome sensitivity by almost a factor of five. Thus the phytochrome system within a green leaf has a considerably greater sensitivity to changes in the environmental light distribution than that in vitro or in etiolated tissue. We wish to point out that this latter point is not usually considered when the phytochrome equilibrium, determined for an etiolated system, is transferred to and used for discussions on green leaves. These considerations may be extended from the "daylight" situation, which represents the natural light environment with the highest red/far-red ratio, to all the various degrees of "shade light" in which the red/far-red ratio continuously decreases. Under these conditions the Pfr/Ptot ratio will be translated continuously towards lower values and hence as can be seen in Fig. 6 towards ever increasing spectral detection sensitivity.

These latter comments refer to the increase in spectral sensitivity of the photostationary state for the phytochrome system in folia with respect to that in non-green tissue or in vitro. However, perhaps surprisingly, it seems that for green tissue, where the light absorption flux by phytochrome is greatly decreased by chlorophyll masking, it has never been demonstrated whether the phytochrome photostationary state is in fact rapidly attained or not. This point is important as it is only in the first case that the phytochrome photoequilibrium will be of physiological importance in green tissue. For phytochrome types in which the $k_{\mathrm{d}}$ may be ignored it is readily shown that the photoequilibrium lifetime, i.e. the $1 / \mathrm{e}$ photoequibration time, is given by $\left(k_{1}+k_{-1}\right)^{-1}$. As these rate constants scale linearly with the light absorption flux, knowledge of these absorption fluxes in a leaf will allow determination of the photoequilibrium lifetime. To this end we have used the spectra of Fig. 5 and the estimated leaf phytochrome content based on the monoclonal antibody determinations of Sharrock and Clack. ${ }^{13}$ If then we consider that the leaf immersed in a "daylight" light environment of quite high intensity $\left(\sim 5 \mu\right.$ Einstein $\left.\mathrm{m}^{-2} \mathrm{~s}^{-1} \mathrm{~nm}^{-1}\right)$ and assuming a quantum efficiency of photoconversion in both directions of 1 , we estimate a rather rapid photoequilibrium lifetime of about $0.5 \mathrm{~s}$. However, published quantum efficiency values, while being rather variable, are much closer to 0.1 
(e.g. about 0.15 for the $\mathrm{Pr} / \mathrm{Pfr}$ conversion and 0.07 for the $\mathrm{Pfr} / \mathrm{Pr}$ conversion $^{4}$ ) and so, in this case, the photoequilibrium lifetime would increase to about $5 \mathrm{~s}$. This is still a short time in physiological terms. This conclusion is rather interesting as it clearly shows that, despite the very low absorption of phytochrome in a leaf, due to its low concentration and optical masking by chlorophylls, photoequilibrium is rapidly attained on a physiological time scale. It is worth noting that, even in a "shade light" environment, the photoequilibrium lifetime may also not be very long, despite the overall decrease in light intensity which may reach two orders of magnitude. This is because, while $k_{1}$ decreases greatly in "shade light" due to the great optical masking of Pr absorption, the photoequilibration time, in these conditions, is determined largely by $k_{-1}$, because it decreases much less. We estimate, for the "shade light" spectral distributions published by Holmes and Smith ${ }^{16}$ and considering, as above, a photochemical photoconversion quantum efficiency of the order of 0.1 , a photoequilibration lifetime in the range of $20-80 \mathrm{~s}$.

\section{References}

$1 \mathrm{H}$. Smith, Phytochromes and light perception by plants - an emerging synthesis, Nature, 2000, 407, 585-591.

2 I. R. Gilbert, P. G. Jarvis and H. Smith, Proximity signal and shade avoidance differences between early and late successional trees, Nature, 2001, 411, 792-795.

3 S. E. Braslavsky, W. Gartner and K. Schaffner, Phytochrome photoconversion, Plant Cell Env., 1997, 20, 700-706.

4 L. C. Sage, Pigment of the Imagination: A History of Phytochrome Research. Academic Press, Inc., San Diego 1992.

5 T. Ohtani and T. Kumagai, Action spectra for the light inhibition of flowering and its reversal in Lemna paucicostata T-101, Plant Cell Physiol., 1980, 21, 135-138.
6 F. M. Garlaschi, G. Zucchelli and R. C. Jennings, Studies on light absorption and photochemical activity changes in chloroplast suspensions and leaves due to light scattering and light filtration across chloroplasts and vegetation layers, Photosynth. Res., 1989, 20, 207220

7 A. Rivadossi, G. Zucchelli, F. M. Garlaschi and R. C. Jennings, The importance of PSI chlorophyll red forms in light harvesting by leaves, Photosynth. Res., 1999, 60, 209-215.

8 M. G. Holmes and L. Fukshansky, Phytochrome photoequilibria in green leaves under polychromatic radiation: a theoretical approach, Plant Cell Env., 1979, 2, 59-65.

9 W. L. Butler, Absorption of light by turbid materials, J. Opt. Soc. Am., 1962, 52, 292-299.

10 A. Rivadossi, G. Zucchelli, F. M. Garlaschi and R. C. Jennings, Light absorption by chlorophyll $a-b$ complexes of photosystem II in a leaf with special reference to LHCII, Photochem. Photobiol., 2004, 80, 492 498

11 J. C. Lagarias, J. M. Kelly, K. L. Cyr and W. O. Smith, Comparative photochemical analysis of highly purified 124 kilodalton oat and rye phytochromes in vitro, Photochem. Photobiol., 1987, 46, 5-13.

12 K. Eichenberg, I. Bäurle, N. Paulo, R. A. Sharrock, W. Rüdiger and E. Schäfer, Arabidopsis phytochromes $\mathrm{C}$ and $\mathrm{E}$ have different spectral characteristics from those of phytochromes A and B, FEBS Lett., 2000, 470, 107-112.

13 R. A. Sharrock and T. Clack, Patterns of expression and normalized levels of the five Arabidopsis phytochromes, Plant Physiol., 2002, 130, $442-456$.

14 P. H. Quail, M. T. Boylan, B. M. Parks, T. M. Short, Y. Xu and D. Wagner, Phytochromes: Photosensory perception and signal transduction, Science, 1995, 268, 675-680.

$15 \mathrm{H}$. Smith and M. G. Holmes, The function of phytochrome in the natural environment - III. Measurement, and calculation of phytochrome photoequilibria, Photochem. Photobiol., 1977, 25, 547550

16 M. G. Holmes and H. Smith, The function of phytochrome in the natural environment - I. Characterization, of daylight for studies in photomorphogenesis and photoperiodism, Photochem. Photobiol., 1977, 25, 533-538. 